\title{
Artigos
}

\section{A compreensão dos docentes sobre a formação em Saúde Coletiva nos cursos de Fonoaudiologia de universidades públicas do Nordeste brasileiro}

Education for public health from the perspective of professors teaching on speech-language-hearing undergraduate programs in public universities in the Northeast of Brazil (abstract: p. 17)

La comprensión de los docentes sobre la formación en salud colectiva en los cursos de fonoaudiología de universidades públicas del Nordeste brasileño (resumen: p. 17)

Maurício Wiering Pinto Telles ${ }^{(a)}$

<mauwiering@gmail.com> (iD

Luiz Roberto Augusto Noro(b)

<luiz_noro@hotmail.com> i (a) Pós-graduando do Programa de Pós-Graduação em Saúde Coletiva (Doutorado), Centro de Ciências da Saúde (CCS), Universidade Federal do Rio Grande do Norte (UFRN).

Avenida Senador Salgado Filho, 1787, Lagoa Nova. Natal RN, Brasil. 59056-000.

(b) Programa de Pós-Graduação em Saúde Coletiva, CCS, UFRN Natal, RN, Brasil.

No Brasil, os cursos de graduação em Fonoaudiologia, com a implantação das Diretrizes Curriculares Nacionais (DCN), ampliaram a oferta de componentes curriculares de Saúde Coletiva, buscando fortalecer a inserção dos estudantes no Sistema Único de Saúde (SUS). Diante disso, este artigo objetiva compreender a formação em Saúde Coletiva dos cursos de Fonoaudiologia em universidades públicas do Nordeste brasileiro, na perspectiva docente. Para a produção de dados foram realizadas entrevistas com sete informantes-chave, e para a análise foram utilizados os referenciais teóricos sobre Saúde Coletiva e atos de currículo. Nos resultados, identificou-se uma compreensão dos docentes sobre a formação em Saúde Coletiva como espaço privilegiado para a formação no contexto do SUS, ainda que tenha sido observada uma heterogeneidade na concepção de Saúde Coletiva. Isso aponta para a necessidade de fomentar estudos que subsidiem a ampliação da inserção dos estudantes no SUS.

Palavras-chave: Fonoaudiologia. Educação Superior. Avaliação educacional. 


\section{Introdução}

A reorientação da formação na Saúde apresenta-se como um dos desafios para este século, visto que o perfil dos profissionais desse setor precisa acompanhar as modificaçóes demográficas e epidemiológicas da população ${ }^{1}$. Em um panorama geral, observa-se que a formação ainda está baseada em um modelo que privilegia a atenção à doença e a cura, de forma fragmentada, e de incipiente integração do conhecimento ${ }^{2}$. Ressalta-se ainda que, nesse cenário, há uma centralidade na formação clínica especializada individual em detrimento de práticas colaborativas, interdisciplinares e interprofissionais ${ }^{3,4}$.

Diante disso, torna-se relevante compreender como os cursos de graduação na área da Saúde no Brasil, dentre esses a Fonoaudiologia, têm orientado a formação profissional. Esse tipo de análise busca favorecer a construção de ferramentas que subsidiem a tomada de decisóes no planejamento acadêmico, na construção dos Projetos Pedagógicos de Curso (PPC) e de matrizes curriculares que objetivem formar profissionais aptos a atuar nos principais problemas de saúde da população e nas necessidades do Sistema Único de Saúde (SUS), conforme sugerem as Diretrizes Curriculares Nacionais (DCN) .

Estudos realizados na Fonoaudiologia têm apontado uma deficiência da formação no SUS, sobretudo no contexto da Atenção Primária à Saúde (APS ${ }^{6,7}$. Essa realidade favorece, inclusive, a baixa inserção do fonoaudiólogo nesse campo de trabalho, além de fragilizar a atuação dos profissionais nos diferentes serviços de saúde ${ }^{7,8}$.

No que concerne à formação em Saúde Coletiva, apesar de não serem encontradas em volume expressivo, pesquisas que abordam a temática têm demonstrado um avanço da Saúde Coletiva na Fonoaudiologia, inclusive na distribuição de componentes curriculares obrigatórios ${ }^{9}$, implicando contribuições para a reorientação profissional. Tais contribuições referem-se à ampliação do olhar do discente sobre a saúde e à realização de vivências no SUS, com oportunidade de trabalhar de forma interdisciplinar ${ }^{10-12}$. Ainda assim, desafios continuam sendo identificados, como a dificuldade de discentes em relacionar os conceitos da Saúde Coletiva com a atuação da Fonoaudiologia e fragilidades na integração ensino-serviço-comunidade ${ }^{10-12}$.

Diante da problemática apresentada, considera-se relevante a análise da formação em Saúde Coletiva nos cursos de Fonoaudiologia. Por isso, partindo do entendimento de que os docentes são atores curriculares implicados na formação ${ }^{13,14}$, este artigo objetiva analisar a compreensão de docentes sobre a formação em Saúde Coletiva em cursos de graduação em Fonoaudiologia de Instituições de Educação Superior (IES) públicas do Nordeste brasileiro. A escolha em estudar o caso dessa região ocorre pela característica da baixa inserção e distribuição de fonoaudiólogos no SUS na localidade ${ }^{15,16}$, contexto que estimula a pesquisa sobre as potencialidades e os limites dos cursos de Fonoaudiologia no que se refere à formação de profissionais com perfil voltado para atuar de acordo com as necessidades da população e do SUS, e de como a Saúde Coletiva tem contribuído para o processo formativo. 


\section{Metodologia}

Pesquisa de abordagem qualitativa, estudo de caso, foi o método utilizado por favorecer a construção de uma etapa exploratória de fenômenos pouco investigados ${ }^{17}$. São sujeitos deste estudo docentes graduados em Fonoaudiologia envolvidos com componentes curriculares de Saúde Coletiva de sete IES públicas do Nordeste: Universidade Federal do Rio Grande do Norte, Universidade Federal da Paraíba, Universidade Federal de Pernambuco, Universidade Estadual de Ciências da Saúde de Alagoas, Universidade Federal de Sergipe campus São Cristóvão, Universidade Federal de Sergipe campus Lagarto, e Universidade Federal da Bahia. Destaca-se que, na região, há oito cursos públicos de Fonoaudiologia. Neste estudo participaram as IES que sinalizaram, quando consultadas, possuir docentes fonoaudiólogos efetivos dedicados aos componentes curriculares de Saúde Coletiva.

Para cada IES participante foi definido um informante-chave, objetivando a realização de entrevistas com indivíduos que possuem conhecimento especial e informação profunda sobre a temática da pesquisa ${ }^{18}$. Na IES que possuía mais de um docente fonoaudiólogo atuando na área de Saúde Coletiva, foi convidado aquele que ocupava ou houvera ocupado cargo de gestão na IES. Em caso de ausência dessa característica era convidado o docente com maior tempo de atuação na IES. Esses critérios buscaram contemplar na pesquisa a participação de professores que tivessem maior experiência e trajetória no curso que lecionam.

O roteiro de entrevistas buscou contemplar questóes que norteassem o docente a relatar a organização dos componentes curriculares de Saúde Coletiva no curso, o conceito de Saúde Coletiva ensinado, a inserção dos estudantes no SUS e as potencialidades e limites na formação em Saúde Coletiva. Das sete entrevistas, duas foram realizadas de forma presencial e cinco a distância por meio da ferramenta de comunicação e videochamadas Skype. As entrevistas foram gravadas em áudio e transcritas, sendo os dados analisados utilizando a Análise Temática de Conteúdo ${ }^{19}$. Para tanto, inicialmente foi realizada uma leitura exaustiva do conjunto das comunicações para imersão no conteúdo, seguida da exploração do material que originou a elaboração de quatro categorias de análise: a) concepção de Saúde Coletiva adotada nos cursos, b) a Saúde Coletiva no currículo dos cursos de Fonoaudiologia das universidades públicas do Nordeste, c) atos de currículo e possibilidade para a formação no SUS e d) potencialidades e limites na formação em Saúde Coletiva segundo os atores curriculares docentes. Posteriormente, o material produzido foi organizado em uma matriz analítica, sendo os dados ordenados conforme as categorias que emergiram no momento da exploração.

Para a interpretação dos dados foram utilizados os referenciais teóricos sobre o conceito de Saúde Coletiva ${ }^{20}$ e sobre atos de currículo ${ }^{13,14}$. Com isso, compreende-se neste estudo a Saúde Coletiva como um campo de saber voltado para a compreensão da saúde e a explicação de seus determinantes sociais, bem como o âmbito de práticas direcionadas prioritariamente para a sua promoção, além de voltadas para a prevenção e o cuidado a agravos e doenças, tomando por objeto a coletividade ${ }^{20}$. Já a compreensão de atos de currículo adotada parte do entendimento de que o currículo é um texto em constante escrita, possuindo caráter relacional e construcionista, sendo os docentes 
(e)

um dos atores/autores curriculares implicados na formação e capazes de dar sentido ao documento curricular, seja seguindo-o conforme escrito ou atribuindo-lhe novas práticas ou significados ${ }^{13,14}$.

O estudo foi aprovado pelo Comitê de Ética em Pesquisa do Hospital Universitário Onofre Lopes da Universidade Federal do Rio Grande do Norte, sob o parecer de número 3.735.493, atendendo às orientações da Resolução 466/12 do Conselho Nacional de Saúde e os participantes assinaram o Termo de Consentimento Livre e Esclarecido.

\section{Resultados e discussão}

Os resultados estão apresentados e discutidos nas quatro categorias de análise identificadas com base no material produzido. Tais categorias buscam sistematizar e analisar a problemática apresentada neste estudo.

\section{Concepção de Saúde Coletiva adotada nos cursos}

Pelo questionamento sobre a abordagem da Saúde Coletiva no curso com os docentes, foi possível identificar uma heterogeneidade de concepçóes. Um dos artifícios utilizados por alguns entrevistados para explicar a concepção adotada foi detalhar o conteúdo programático trabalhado nos componentes curriculares:

Eu sou adepta ao Tratado de Saúde Coletiva, alguns manuais do Ministério. Sou da formação da época de enfermagem de Ricardo Ayres, mas eu trabalho muito o Tratado de Saúde Coletiva. (P6)

$\mathrm{Na}$ disciplina de saúde e sociedade eu tento trazer mais conceitos da compreensão do fenômeno saúde-doença, e aí tanto pela abordagem das ciências sociais em saúde, dos determinantes sociais da saúde como também pelo olhar epidemiológico [...] (P2)

Um dos pontos destacados por docentes é o de que o conceito de Saúde Coletiva deve ser diferenciado do conceito de saúde pública. Apesar de nem todos se aprofundarem no tema ou não esclarecerem em que os conceitos se diferenciam, professores entrevistados destacam que esse é um ponto que deve ser reforçado com os estudantes.

Os conceitos de saúde pública e Saúde Coletiva e a diferenciação entre ambos não se trata de um consenso ${ }^{21,22}$. Contudo, identifica-se na literatura que a Saúde Coletiva surge no final da década de 1970 em contraposição à medicina preventiva, à saúde pública institucionalizada e ao liberalismo no campo médico ${ }^{23}$. Essa contraposição soma-se à luta pela redemocratização do Estado, pautando-se em uma Reforma Sanitária Brasileira (RSB) que contemplasse uma reforma social ${ }^{24}$. Pode-se dizer, portanto, que a Saúde Coletiva e a RSB possuem emergência e história de constituição ligada a uma modificação no olhar sobre a saúde e sobre a organização do sistema de saúde ${ }^{25}$. 
No entanto, o que tem sido identificado por autores que participaram da construção da Saúde Coletiva e da RSB é que há um movimento de distanciamento entre elas. O processo da RSB pode ter sofrido imobilismos e ajustes conservadores, com atores que participaram dessa construção adotando posicionamentos que criam barreiras para a construção de um sistema universal ${ }^{25}$. Esses fenômenos podem contribuir também na explicação sobre os rumos da Saúde Coletiva, que tem se direcionado para um caminho de restauração da saúde pública hegemônica ${ }^{25}$.

Percebe-se, então, que a dificuldade dos entrevistados em definir a Saúde Coletiva converge com os dilemas vivenciados pelo campo, sobretudo no que concerne à sustentabilidade do processo da RSB. Além disso, esse desconhecimento ou essa insegurança pode estar relacionado com a característica da Saúde Coletiva que, apesar de legitimada e institucionalizada, é um campo relativamente novo, com pluralidades e aberto a transformaçốes ${ }^{22,26}$.

Outro elemento apresentado pelos entrevistados é a presença de um tripé epistemológico composto pela epidemiologia, ciências sociais e humanas e política, planejamento e gestão em saúde. A presença desse tripé, ainda, foi uma das formas utilizadas para tentar diferenciar a Saúde Coletiva da saúde pública:

Esse olhar da Saúde Coletiva eu tento trazer naquele tripé: na epidemiologia social, desse lado social, do planejamento em saúde e não apenas voltado para os serviços de saúde, pra aquela saúde pública limpa e seca. (P7)

Eu venho trabalhando muito na perspectiva de haver um entendimento da Saúde Coletiva enquanto um campo teórico e de práticas. [...] baseado no tripé do campo epistêmico da Saúde Coletiva que é do complemento da política, planejamento e gestão e avaliação da saúde, das ciências sociais da saúde e epidemiologia. (P2)

Essa perspectiva explicitada demonstra os instrumentos de trabalho utilizados pela Saúde Coletiva. Nesse sentido, compreende-se que esse campo se utiliza da epidemiologia social ou crítica, priorizando os estudos sobre a determinação social, aliados aos conhecimentos das ciências sociais e da prática do planejamento estratégico e da gestão democrática ${ }^{21}$.

Apesar dessa diferenciação, observou-se também em alguns discursos uma abordagem conceitual da Saúde Coletiva como algo vinculado apenas a políticas ou programas de saúde no âmbito do SUS, ou a uma prática profissional amparada no conceito ampliado de saúde:

[...] Mesmo não sendo a Saúde Coletiva aplicada para fono eu já tento trabalhar o raciocínio para fonoaudiologia porque a gente está no curso de fono e eu não posso simplesmente falar da enfermagem, a gente tem que falar da fono mesmo. Aí trago também Nasf, PICs, PSE, Brasil Sorridente, todos os programas [...] .(P6) 
É uma Saúde Coletiva ampla, onde os alunos [...] são formados para serem sujeitos generalistas, humanistas, éticos, reflexivos, críticos. [...] a gente busca fazer um trabalho de promoção dentro dos preceitos da Saúde Coletiva [...], onde veja o ser humano de uma maneira integral e não só aquele local que ele diz estar acometido. (P3)

Apenas um dos entrevistados referiu utilizar, como abordagem da Saúde Coletiva, a identificação das necessidades de saúde e atuação sobre os determinantes sociais por meio do trabalho prioritário com a promoção da saúde e prevenção dos danos e agravos e com a reabilitação. Apesar disso, esse sujeito relata que essa é uma concepção que pode ser diferente em seu curso a depender do docente que esteja à frente do componente curricular.

\section{A Saúde Coletiva no currículo dos cursos de Fonoaudiologia das universidades públicas do Nordeste}

Partindo do pressuposto de que a formação é um processo que se depara com uma incompletude infinita, entende-se que essa favorece o saber-refletir, saber-fazer, saber-ser, mediado pelos saberes da experiência, qualificando o Ser a aprender a aprender, a reaprender e a desaprender, refletindo criticamente sobre a própria experiência formativa ${ }^{27} . \mathrm{Na}$ educação, o dispositivo que mediatiza a formação é o currículo, no qual são explicitados os conhecimentos eleitos como formativos ${ }^{13}$.

Ao compreender que a formação não é resultado de ações exterodeterminadas, admitese que o currículo não representa um simples texto-guia aplicado na prática educacional ${ }^{14}$. Por isso, o entendimento sobre atos de currículo forjado por Macedo ${ }^{13,14}$ contribuirá na análise de que o currículo é um texto em constante escrita, possuindo caráter relacional e construcionista, e que possui inflexóes de sujeitos curriculantes, isto é, que constroem e póem em prática o currículo. Nessa perspectiva, compreender a estrutura e a dinâmica curriculares por meio dos atores curriculares pode viabilizar a identificação de elementos que não são possíveis de ser analisados nos documentos institucionais.

Dos sete cursos participantes da pesquisa, apenas a Aprendizagem Baseada em Problemas (ABP) é um PPC e uma matriz curricular orientados por metodologia ativa. Nesse curso, a Saúde Coletiva ocorre de forma transversal nos módulos de aprendizagem, com algumas disciplinas durante o curso.

Nos demais cursos, o PPC e a matriz curricular são organizados de forma tradicional, isto é, disciplinar. Contudo, três cursos possuem componentes curriculares de Saúde Coletiva em quase todos os semestres, sendo que, em um dos cursos, professores não pertencentes ao quadro do departamento de Fonoaudiologia lecionam disciplinas da área durante metade do curso. 
[...] eles têm disciplinas teóricas no [Instituto] do primeiro ao quinto semestre. [...] Aí no sexto semestre eles têm uma disciplina que é ofertada por a gente.

[...] Então do primeiro ao sexto na verdade eles têm várias disciplinas teóricas e a partir do sétimo eles começam o [...] estágio I e II, sétimo Saúde Coletiva I, oitavo estágio em Saúde Coletiva II. Atualmente a disciplina do nono que é práticas I a gente fica responsável [...]. (P1)

Ela [a disciplina de Saúde Coletiva III] é no quarto período, porque [as disciplinas de Saúde Coletiva ocorrem] no segundo, terceiro e quarto [períodos]. Porque no quinto [período] a gente já começa os estágios. (P5)

Os currículos disciplinares, que possuem inspiração no processo de trabalho taylorista e fordista, possuem a capacidade de orientar a formação de profissionais de saúde em uma perspectiva de valorização da aprendizagem tecnicista em detrimento da formação social e humana dos profissionais ${ }^{28}$. Essa perspectiva proporciona poucas oportunidades de aprendizagem e de prática interdisciplinar, sem o devido preparo para o trabalho em equipe, ainda que haja essa exigência na atuação profissional ${ }^{28}$. Pode-se afirmar inclusive que, ao ser inserida como componente curricular, a Saúde Coletiva funciona como um apoio à aproximação, ainda que incipiente, dos estudantes à realidade social.

Nos cursos de currículo tradicional, dedicam-se os primeiros componentes curriculares para a abordagem teórica da Saúde Coletiva e, ao final do curso, ofertam estágios na área. Apenas um curso com currículo tradicional realiza a inserção no SUS desde o segundo período. No entanto, alguns cursos buscam realizar visitas técnicas em instituiçôes ao longo dos componentes teóricos para dirimir o distanciamento com a prática.

Em cada temática dessa a gente trabalha quais as políticas que dão o arcabouço, o que de fato a gente relaciona com a fonoaudiologia e como a fonoaudiologia se insere dentro dessa política e a rede de atenção que dá suporte. [...] eu faço uma visita técnica à [instituição] nessa disciplina. (P5)

A gente já foi para a Academia da Saúde, [...] para Centro de Referência e Reabilitação [...]. Esse semestre agora [...] está agendado para daqui a 15 dias [...] acho que é o Centro de Referência e Reabilitação[...] que é um que atende microcefalia [...]. (P6)

A existência do currículo e da ação formativa tem por objetivo a alteração, a mudança, a modificação, no processo de constituição do Ser e na construção de identidades ${ }^{27}$. No entanto, o tradicionalismo, representado muitas vezes pela disciplinaridade e pelo distanciamento entre teoria e prática, constitui-se como limite para uma experiência significativa no processo formativo dos estudantes de Fonoaudiologia. Essa problemática tem sido objeto de estudo de diferentes autores que apontam a necessidade de estimular a educação pelo trabalho ${ }^{29}$, a integração ensino-serviço-comunidade ${ }^{30}$ e a utilização de metodologias ativas no processo de ensino-aprendizagem ${ }^{31}$. 


\section{Atos de currículo e possibilidades para a formação no SUS}

Das sete IES estudadas, cinco realizaram reformulação curricular recente. Essas reformas tiveram uma perspectiva de adequação do PPC às DCN, com ampliação da oferta de componentes curriculares em Saúde Coletiva, visando maior inserção no SUS. Contudo, algumas limitações ainda não foram superadas.

O que foi priorizado? Que tivesse Saúde Coletiva como um eixo transversal do primeiro ao último ano. [...] a gente tem um eixo que [...] chama Saúde e Sociedade, que vai do primeiro ao último ano do curso, e isso serve para todos os cursos da área da Saúde, exceto Medicina. (P4)

No [currículo] anterior os atendimentos de Saúde Coletiva no segundo ano estavam mais na UBS e com essa reformulação a gente conseguiu junto à Secretaria Municipal de Saúde, [...] que os nossos alunos tivessem a prática, o aprendizado, a formação também nas maternidades, hospital, também na Caps e no Cerest. (P3)

No que concerne à integração dos conteúdos dos componentes curriculares das ciências básicas com a Saúde Coletiva e com a clínica fonoaudiológica, foi possível observar que permanece como um limite. Essa dissociação entre os conhecimentos biológicos e da clínica da compreensão dos fenômenos e políticas sociais também foi encontrada em um estudo sobre projetos pedagógicos do curso de Nutrição ${ }^{32}$, reafirmando os desafios existentes no âmbito da formação em Saúde para serem proporcionados em uma integração dos saberes. No entanto, estudos sobre a construção de currículos integrados na área da Saúde têm sido elaborados com o objetivo de demonstrar os limites do modelo curricular que separa as ciências básicas das clínicas, ainda largamente adotado nos cursos de graduação, e apontando estratégias pedagógicas que podem favorecer a integração dos conteúdos ${ }^{33,34}$.

Oficialmente não [existe integração dos conteúdos]. A gente parte disso quando a gente começa a ofertar só no sexto semestre. Quando chega em Saúde Coletiva em Fonoaudiologia, a gente parte da trajetória que eles fizeram até o quinto. Então a gente já entende que eles já discutiram política, uma serie de conceitos [...] (P1)

Houve uma tentativa no ano de 2017 da gente fazer [uma estratégia de integração dos conteúdos]. Aí dentro do NDE a gente começou a juntar as ementas dessas disciplinas, inclusive propor até avaliações que envolvessem uma avaliação interdisciplinar [...]. Mas depois, devido a um problema conjuntural que o curso passou [...] os professores entregaram a função do NDE e também a chefia de departamento [...] saiu do cargo, e essa ação foi quebrada. (P2) 
Nesse último trecho, observa-se que as tentativas de integração dos conteúdos são também objetos de disputa de ideias dentro do corpo docente. Essa perspectiva evidencia que, no processo formativo, o currículo como instrumento de mediação não está alheio às tensões ou conflitos. Isso quer dizer que, apesar da existência de propostas como as DCN ou de outros documentos que visam orientar os processos formativos, a dinâmica curricular é composta por atores curriculares que, com suas ideologias, interesses e vivências, buscam de certa forma modificar, alterar ou inflexionar o currículo e, consequentemente, a formação dos profissionais de saúde ${ }^{13,14}$.

Adicionalmente, a formação interdisciplinar e interprofissional também não é uma realidade para os cursos de Fonoaudiologia do Nordeste. Apenas duas docentes relataram que durante o percurso acadêmico o estudante está exposto a uma experiência interdisciplinar ou interprofissional, sobretudo nos componentes curriculares de Saúde Coletiva. Um dos cursos possui, inclusive, componentes curriculares teóricos que são ofertados de forma obrigatória para diferentes cursos da área da Saúde no seu PPC.

[...] A gente visa isso, que o atendimento não seja só de fono, as práticas sejam só de fisio, tudo separado não, a gente procura fazer projetos interdisciplinares e que a aula seja também interdisciplinar. (P3)

Então, na Saúde e Sociedade I, todos os cursos têm turmas mistas: fono, fisio, TO, e enfermagem. [...] Nos outros campos de estágio, apesar deles, por exemplo, na clínica eles estão no CER [...] então todos os cursos estão nesse CER, mas é muito difícil de que ocorram práticas interprofissionais. [...]Mas na Saúde Coletiva a gente teve o movimento de fazer acontecer, mesmo com toda dificuldade. (P4)

Para Honoré35, a formação interprofissional "se trata de poner en marcha para vencer la separación entre vida profissional, vida personal y las diversas dimensiones de la vida social" (p. 157). Tal formação interprofissional, bem como a interdisciplinar, é um dos principais desafios colocados para as graduaçôes em Saúde neste século ${ }^{1}$. O perfil demográfico, epidemiológico e aqueles relacionados ao perfil social da população têm requerido cada vez mais que as práticas nos serviços profissionais sejam orientadas pelo princípio colaborativo e da interprofissionalidade, em que os profissionais com seus saberes devem intervir nos problemas de saúde em equipe, com o cuidado compartilhado.

Internacionalmente, cursos da área da Saúde têm construído currículos que contemplam a Educação Interprofissional (EIP) desde os anos iniciais, buscando aproximar os estudantes do conhecimento das singularidades e complementaridades das diferentes áreas de atuação profissional, por meio do ensino-aprendizagem que favoreça experiências educacionais compartilhadas, bem como dos sistemas de saúde ${ }^{36,37}$. Essa organização curricular deve favorecer a preparação dos profissionais para fornecer cuidados seguros e eficazes nos sistemas de saúde que se encontram cada vez mais complexos ${ }^{37}$. Apesar dos avanços, nota-se que essa realidade ainda não abrange a totalidade da formação dos profissionais da saúde, como no caso dos cursos estudados 
nesta pesquisa. Ainda assim, experiências interprofissionais e interdisciplinares que alguns docentes têm proporcionado aos estudantes em projetos e práticas, principalmente na Saúde Coletiva, demonstram que novos caminhos podem estar sendo construídos para a superação da lógica formativa uniprofissional.

Os cursos participantes da pesquisa possuem inserção no SUS, sobretudo no âmbito da APS. Essa inserção, no entanto, ocorre em sua maioria nos estágios em Saúde Coletiva nos anos finais do curso, bem como em hospitais universitários para os estágios de outras áreas da Fonoaudiologia, além das clínicas-escolas conveniadas ao SUS. Esse convênio, no entanto, ocorre apenas em três das clínicas-escolas dos cursos estudados, sendo as outras quatro mantidas pela universidade como um serviço gratuito, mas sem vínculo com a rede pública de saúde.

\section{Potencialidades e limites da formação em Saúde Coletiva segundo os atores curriculares docentes}

Os entrevistados relataram que o corpo docente que ministra os componentes curriculares de Saúde Coletiva tem, em sua maioria, uma boa formação na área e é dedicado na realização do seu trabalho. A possibilidade de ter contato com professores de outras categorias profissionais no ensino de Saúde Coletiva também foi pontuado como algo que pode acrescentar na aprendizagem do estudante.

Outro ponto forte é a gente ter uma equipe muito comprometida, eu acho que o nosso grupo [de professores da área de Saúde Coletiva] é de fato muito especial, a gente além de ter uma formação boa na área de Saúde Coletiva, nós temos práticas diferentes que se somam. (P5)

Potencialidades eu acho que a variação de formação que os professores têm. A universidade por ter essa questão de ter estrutura em núcleo, consegue ter vários profissionais de várias áreas que podem ter olhares totalmente diferentes e somar [...]. (P4)

Os professores do ensino superior, como sujeitos implicados na formação de futuros profissionais e que possuem seus desejos, suas crenças, suas justificativas e suas ideologias, são atores constituintes dos atos de currículo ${ }^{14}$. Sendo assim, além de suas intencionalidades, é de fundamental importância também para os atos de currículo a sua formação pedagógica para compreender o seu papel como mediador de aprendizagem ${ }^{38}$. Com isso, um corpo docente comprometido e capacitado, como relatado pelos entrevistados, deverá contribuir na formação dos futuros fonoaudiólogos, embora seja necessária a avaliação dos demais atores curriculares para que essas características sejam confirmadas.

Contudo, também foram apontadas fragilidades por entrevistados relacionadas à prática docente. Uma das dificuldades foi a do trabalho em equipe no núcleo de professores que ofertam os componentes curriculares de Saúde Coletiva, sobretudo no departamento de fonoaudiologia. Esse contexto fez que, inclusive, houvesse um ambiente de tensão e rupturas no espaço de trabalho, situação relatada por três entrevistados. 
Outro limite se refere à quantidade reduzida de professores para atuar no campo da Saúde Coletiva. Esse cenário provoca uma sobrecarga no trabalho, trazendo como conseqüência a baixa oferta de extensão e pesquisa na área.

[...] Limites existem desde a questão do RH, do número de professores, tem a ver com a carga horária desses professores [...]. Os professores que entraram no concurso mais recente são professores de 20 horas e sem perspectiva de ampliaçãoo de carga horária [...]. (P4)

[...] eu considero também como um entrave a questão de não ter preceptor. $\mathrm{O}$ professor é supervisor, é orientador, acompanha os alunos, dá a parte teórica. (P7)

A sobrecarga no trabalho docente é uma das consequências das contrarreformas que afetaram políticas sociais nas áreas da Educação, Saúde e Assistência Social ${ }^{39}$. Essas contrarreformas, impulsionadas pelo neoliberalismo, acarretaram o desinvestimento das universidades públicas ${ }^{39}$, implicando, inclusive, a redução da contratação de novos professores.

O aumento das atribuiçốes acontece também devido à ausência de preceptores no SUS, fazendo que o docente tenha de assumir esse papel. Um dos motivos apontados para a ausência de preceptores é a baixa cobertura da assistência fonoaudiológica na rede. Além disso, por inexistência da preceptoria e pelas limitaçóes do currículo, a maioria dos cursos possui inserções pontuais nos serviços de saúde.

Segundo os entrevistados a problemática referente à inserção no SUS é acentuada em determinados cursos porque algumas das clínicas-escolas não são conveniadas com o SUS. Sendo assim, a formação nas outras áreas da Fonoaudiologia acaba por não possuir ou possuir pouca inserção nesse sistema.

Se tivesse preceptores no município seria bem melhor, porque a atuação do professor enquanto supervisor, assim do professor supervisor e preceptor porque tanto seria bom pra rede [...] como também para os alunos porque eles iriam olhar um profissional que está atuando.(P2)

[...] a questão de que por não ser do SUS, não tem profissionais técnicos. Então aquele usuário que está em tratamento e acompanhamento quando chega férias de aluno o tratamento é suspenso [...]. (P7)

A inserção nos campos de estágio no SUS, em contrapartida, é facilitada pela boa relação construída pelos docentes de Saúde Coletiva com os profissionais dos serviços de saúde. Apesar das dificuldades, essa relação acaba por proporcionar espaços de aprendizagem importantes para o futuro fonoaudiólogo. Nesse quesito, depreende-se que a integração ensino-serviço tem proporcionado um espaço de ensino-aprendizagem 
que transforma o olhar do estudante e a prática dos profissionais das equipes de saúde, provocando reflexões sobre necessidades de mudança na Atenção à Saúde e construindo novas formas de intervir na realidade ${ }^{30}$.

Como potencialidades na perspectiva dos entrevistados, a metodologia utilizada, seja nos componentes curriculares, seja na própria estrutura curricular, a carga horária ocupada no currículo e as possibilidades de extensão e pesquisa na área também foram destacadas. Segundo relatos, essa formação permite que o egresso do curso possa ter uma compreensão diferenciada sobre a saúde e sobre a atuação no SUS.

[...] quando eu vejo na minha formação e vejo na formação deles, é muito melhor. Eles têm uma disciplina aplicada a fono, eles têm um estágio supervisionado, porque, claro, o contexto que eu me formei e o contexto de hoje é bem diferente. (P6)

Acho que uma potencialidade é a questão da sensibilização deles pra uma visão diferente de sociedade. Então, isso é muito trabalhado pra que eles compreendam que existem aspectos macropolíticos que determinam o processo saúde-doença. (P2)

Um dos pontos citados sobre as fragilidades para a formação em Saúde Coletiva foi a ausência da integração dos conteúdos e da formação interdisciplinar e interprofissional, limitações já identificadas na seção anterior. Essa ausência de integração acontece ainda dentro da clínica, quando as áreas de conhecimento da Fonoaudiologia atuam de forma independente, e dentro da Saúde Coletiva, quando departamentos que não são de Fonoaudiologia ofertam componentes curriculares.

Nessa reforma houve uma superfragmentação, superespecialização no currículo. Aumentou muito carga horária, transformou-se, na minha leitura, em cinco cursos por área que não dialogam entre si. (P1)

Nos outros campos de estágio, apesar deles, por exemplo, na clínica eles estão no CER, nós temos um CER III, então todos os cursos estão nesse CER, mas é muito difícil de que ocorram práticas interprofissionais mesmo estando no mesmo cenário. (P4)

A ausência da integração dos conteúdos é influenciada pela falta de uma formação interdisciplinar e interprofissional na Fonoaudiologia. Essa questão fica mais evidente quando os estudantes e professores dividem o mesmo espaço de estágio e práticas de outros cursos nos serviços de saúde, mas mantêm o planejamento e a execução das ações desarticuladas. 


\section{Considerações finais}

Nos resultados deste artigo, foi possível identificar elementos convergentes no que concerne à compreensão da formação em Saúde Coletiva dos docentes de Fonoaudiologia das IES públicas do Nordeste, ainda que tenha sido observada uma heterogeneidade na concepção de Saúde Coletiva adotada pelos atores curriculares entrevistados. Tais elementos relacionam-se com a visualização da Saúde Coletiva como um espaço privilegiado de formação dos estudantes no contexto do SUS, aproximando-os das necessidades desse sistema e da saúde da população. Sendo assim, pode-se dizer que a inserção da Saúde Coletiva em componentes curriculares, conduzidos por professores comprometidos com o processo de ensino-aprendizagem dos estudantes, tem potencializado a formação do fonoaudiólogo que atuará no SUS.

Contudo, são identificadas também fragilidades pelos docentes na formação em Saúde Coletiva. Isso porque a aprendizagem e a prática interprofissional e interdisciplinar, bem como a integração dos conteúdos, ainda se encontram incipientes nos cursos em que atuam. A modificação desse cenário, no entanto, tem sido perseguida pelos docentes, ainda que sejam encontradas dificuldades para a sua efetivação, como a resistência de outros atores curriculares ou a ausência de preceptores fonoaudiólogos no SUS.

O principal desafio para o desenvolvimento deste trabalho foi a impossibilidade de a produção de dados ter sido realizada integralmente de forma presencial, o que inviabilizou a organização adequada dos ambientes para as entrevistas. No entanto, considera-se que essa realidade não interferiu significativamente na qualidade dos dados produzidos. Por fim, ressalta-se que novos estudos que abordem a temática da formação em Saúde Coletiva nos cursos de Fonoaudiologia devem ser realizados, utilizando-se também de outras abordagens metodológicas para que haja uma ampliação dos cenários e sujeitos de estudo. Essas investigações poderiam contemplar questôes como a percepção de outros atores curriculares e o que se propóe nos PPC sobre a formação no SUS e em Saúde Coletiva, bem como os projetos de formação em disputa na formação dos fonoaudiólogos. Dessa forma, em conjunto, tais estudos possuiriam a potencialidade de fundamentar processos de revisão de PPC e colaborações interinstitucionais que fortaleçam o campo da Saúde Coletiva na Fonoaudiologia e de experiências formativas vinculadas às necessidades do SUS e da população. 


\section{Contribuições dos autores}

Todos os autores participaram ativamente de todas as etapas de elaboração do manuscrito.

\section{Conflito de interesse}

Os autores não têm conflito de interesse a declarar.

\section{Direitos autorais}

Este artigo está licenciado sob a Licença Internacional Creative Commons 4.0, tipo BY (https://creativecommons.org/licenses/by/4.0/deed.pt_BR).

\section{(cc) BY}

\section{Editor}

Antonio Pithon Cyrino

Editor associado

Pedro José Santos Carneiro Cruz

\section{Submetido em}

$12 / 10 / 20$

Aprovado em

09/03/21

\section{Referências}

1. Frenk J, Chen L, Bhutta ZA, Cohen J, Crisp N, Evans T, et al. Health professionals for a new century: transforming education to strengthen health systems in an interdependent world. Lancet. 2010; 376(9756):1923-58.

2. Machado CDB, Wuo A, Heinzle M. Educação médica no Brasil: uma análise histórica sobre a formação acadêmica e pedagógica. Rev Bras Educ Med. 2018; 42(4):66-73.

3. Poletto PR, Jurdi APS. A experiência de revisão das matrizes curriculares em um projeto pedagógico inovador: caminhos para fortalecer a educação interprofissional em Saúde. Interface (Botucatu). 2018; 22(2):1777-86.

4. Passos E, Carvalho YM. A formação para o SUS abrindo caminhos para a produção do comum. Saude Soc. 2015; 24(1):92-101.

5. Brasil. Ministério da Educação. Conselho Nacional de Educação. Resolução CNE/ CES 5, de 19 de Fevereiro de 2002. Institui diretrizes curriculares nacionais do curso de graduação em fonoaudiologia. Diário Oficial da União [Internet]. 4 Mar 2002 [citado 26 Set 2020]; sec 1, p. 12. Disponível em: http://portal.mec.gov.br/cne/arquivos/pdf/ CES052002.pdf

6. Lima TFP, Acioli RM. A inserção da fonoaudiologia na atenção primária do Sistema Único de Saúde. In: Silva VL, Lima MLLT, Lima TFP, Advíncula KP, organizadores. A prática fonoaudiológica na Atenção Primária à Saúde. São José dos Campos: Pulso Editorial; 2013. p. 25-42. 
7. Zanin LE, Albuquerque IMN, Melo DH. Fonoaudiologia e estratégia de saúde da família: o estado da arte. Rev Cefac. 2015; 17(5):1674-88.

8. Molini-Alvejonas DR, Aboboreira MS, Couto MIV, Samelli AG. Insertion and performance of speech-language pathology and audiology in family health support centers. CoDAS. 2014; 26(2):148-54.

9. Peixoto MVS, Carvalho S, Dourado ADCM, Gonçalves TP, Cerqueira CS. PET-GraduaSUS: contribuiçôes para mudanças curriculares na graduação em fonoaudiologia na Universidade Federal de Sergipe. In: Trenche MCB, Padovani M, Anhoque CF, Garcia VL, organizadores. Políticas indutoras: formação profissional em fonoaudiologia. São José dos Campos: Pulso Editorial; 2020. p. 156-71.

10. Telles MWP, Arce VAR. Formação e PET-Saúde: experiências de estudantes de fonoaudiologia na Bahia. Rev CEFAC. 2015; 17(3):695-706.

11. Correia TM, Telles MWP, Araújo MVR. A formação em saúde coletiva na visão de estudantes de graduação em fonoaudiologia da UFBA. Disturb Comun. 2018; 30(4):679-87.

12. Arakawa AM, Sitta EI, Caldana ML, Machado MAMP. Gestão em saúde: o aprendizado e a formação acadêmica de estudantes de graduação. Rev CEFAC. 2013; 15(4):947-56.

13. Macedo RS. Atos de currículo e formação: o príncipe provocado. Rev Teias. 2012; 13(27):67-74.

14. Macedo RS. Atos de currículos: uma incessante atividade etnometódica e fonte de análise de práticas curriculares. Curriculo Sem Front. 2013; 13(3):427-35.

15. Viégas LHT, Meira TC, Santos BS, Mise YF, Arce VAR, Ferrite S. Speech, language and hearing services in primary health care in Brazil: an analysis of provision and an estimate of shortage, 2005-2015. Rev CEFAC. 2018; 20(3):353-62.

16. Santos JAP, Arce VAR, Magno LD, Ferrite S. Provision of speech, language and hearing services in the public municipal healthcare network in the state capitals of northeast Brazil. Audiol Commun Res. 2017; 22:e1665.

17. Yin RK. Estudo de caso: planejamento e métodos. 2a ed. Porto Alegre: Bookman; 2001.

18. Bisol CA. Estratégias de pesquisa em contextos de diversidade cultural: entrevistas de listagem livre,entrevistas com informantes-chave e grupos focais. Estud Psicol (Campinas). 2012; 29 Supl 1:719-26.

19. Minayo MCS. O desafio do conhecimento: pesquisa qualitativa em saúde. 14a ed. São Paulo: Hucitec; 2014.

20. Vieira-da-Silva LM, Paim JS, Schraiber LB. O que é Saúde Coletiva. In: Paim JS, Ameida-Filho N, organizadores. Saúde coletiva: teoria e prática. Rio de Janeiro: Medbook; 2014. p. 3-12.

21. Souza LEPF. Saúde pública ou saúde coletiva? Espaço Saude. 2014; 15(4):7-21.

22. Osmo A, Schraiber LB. O campo da saúde coletiva no Brasil: definiçốes e debates em sua constituição. Saude Soc. 2015; 24(1):205-18.

23. Vieira-da-Silva LM. Conclusóes - Saúde coletiva: espaço de relaçôes ou campo em consolidação? Salvador, Rio de Janeiro: EDUFBA, Fiocruz; 2018. p. 223-31.

24. Paim JS. Reforma sanitária brasileira: contribuição para a compreensão e crítica. Salvador, Rio de Janeiro: EDUFBA, Fiocruz; 2008. 
25. Paim JS. A reforma sanitária brasileira e a saúde coletiva: concepçóes, posiçóes e tomadas de posição de intelectuais fundadores. In: Vieira-da-Silva LM, organizador. O campo da saúde coletiva: gênese, transformaçôes e articulaçóes com a Reforma Sanitária Brasileira. Salvador, Rio de Janeiro: EDUFBA, Fiocruz; 2018. p. 191-221.

26. Nunes ED. La salud colectiva en Brasil: analizando el proceso de institucionalización. Salud Colect. 2016; 12(3):347-60.

27. Macedo RS. Compreender/mediar a formação: o fundante da educação. Brasília: Pulso Editorial; 2010.

28. Figueiredo GO, Orrillo YAD. Currículo, política e ideologia: estudos críticos na educação superior em saúde. Trab Educ Saude. 2020; 18(1):e0024880.

29. Fonseca GS, Junqueira SR, Zilbovicius C, Araujo ME. Educação pelo trabalho: reorientando a formação de profissionais da saúde. Interface (Botucatu). 2014; 18(50):571-83.

30. Vendruscolo C, Ferraz F, Prado ML, Kleba ME, Reibnitz KS. Integração ensino-serviço e sua interface no contexto da reorientação da formação na saúde. Interface (Botucatu). 2016; 20(59):1015-25.

31. Souza C, Iglesias A, Pazin-Filho A. Estratégias inovadoras para métodos de ensino tradicionais - aspectos gerais. Medicina (Ribeirão Preto). 2014; 47(3):284-92.

32. Recine E, Sugai A, Monteiro RA, Rizzolo A, Fagundes A. Saúde coletiva nos cursos de nutrição: análise de projetos político-pedagógicos e planos de ensino. Rev Nutr. 2014; 27(6):747-60.

33. Brauer DG, Ferguson KJ. The integrated curriculum in medical education: AMEE Guide No. 96. Med Teach. 2015; 37(4):312-22.

34. Quintero GA, Vergel J, Arredondo M, Ariza M-C, Gómez P, Pinzon-Barrios A-M. Integrated medical curriculum: advantages and disadvantages. J Med Educ Curric Dev. 2016; 3(3):133-7.

35. Honoré B. Para uma teoria de la formación. Madrid: Narcea, S.A. de Ediciones; 1980.

36. Illingworth $\mathrm{P}$, Chelvanayagam $\mathrm{S}$. The benefits of interprofessional education 10 years on. Br J Nurs. 2017; 26(14):813-8.

37. Yancey NR, Cahill S, McDowell M. Transformation in teaching-learning: emerging possibilities with interprofessional education. Nurs Sci Q. 2018; 31(2):126-30.

38. Freitas MAO, Cunha ICKO, Batista SHSS, Rossit RAS. Docência em saúde: percepçốes de egressos de um curso de especialização em Enfermagem. Interface (Botucatu). 2016; 20(57):427-36.

39. Lima KRS. Educação superior em tempos de ajustes neoliberais e regressão de direitos. Rev Katalysis. 2019; 22(3):525-35. 
With the implementation of the National Curriculum Guidelines in Brazil, speech therapy degree courses have expanded the provision of modules on public health, aiming to promote the incorporation of students into Brazilian National Health System (SUS). The aim of this article was to understand education for public health from the perspective of professors teaching on speech-language-hearing undergraduate programs in public universities in the Northeast of Brazil. We conducted interviews with seven key informants. The data was analyzed in the light of the relevant literature on public health and "curriculum acts". Although different conceptions of public health were observed, the findings show that professors understand that education for public health is uniquely positioned to promote education in the context of the SUS. The findings point to the need to promote studies that support the incorporation of students into the SUS.

Keywords: Speech therapy. Higher education. Educational assessment.

En Brasil, los cursos de graduación en fonoaudiología, con la implantación de las Directrices Curriculares Nacionales, ampliaron la oferta de componentes curriculares de salud colectiva, buscando fortalecer la inserción de los estudiantes en el Sistema Brasileño de Salud (SUS). Llevando ese hecho en consideración, este artículo tiene el objetivo de comprender la formación en salud colectiva de los cursos de fonoaudiología en universidades públicas del Nordeste brasileño, desde la perspectiva docente. Para la producción de datos se realizaron entrevistas con siete informantes clave y para el análisis se utilizaron los referenciales teóricos sobre salud colectiva y actos de currículo. En los resultados, se identificó una comprensión de los docentes sobre la formación en salud colectiva como espacio privilegiado para la formación en el contexto del SUS, aunque se haya observado una heterogeneidad en la concepción de salud colectiva. Eso señala la necesidad de fomentar estudios que subsidien la ampliación de la inserción de los estudiantes en el SUS.

Palavras-chave: Fonoaudiología. Educación superior. Evaluación educativa. 